\title{
Arterial stiffness and cognitive dysfunction in alzheimer and parkinson's diseases
}

\author{
Yoon-Sang Oh, Dong-Woo Ryu, Jee-Eun Lee, Joong-Seok Kim \\ Department of Neurology, College of Medicine, The Catholic University of Korea, Seoul, Republic of Korea
}

Article Info

\section{Article Notes}

Received: April 28, 2017

Accepted: May 15, 2017

\section{${ }^{*}$ Correspondence:}

Joong-Seok Kim, M.D., Ph.D.

Department of Neurology, Seoul St. Mary's Hospital, The

Catholic University of Korea

222, Banpo-daero, Seocho-gu, Seoul, 06591 Republic of Korea

Phone: +82-2-2258- 6078

Fax: +82-2-599-9686

Email: neuronet@catholic.ac.kr

(c) $2017 \mathrm{Kim}$ JS. This article is distributed under the terms of the Creative Commons Attribution 4.0 International License

\section{Keywords}

Arterial stiffness

Cognitive dysfunction

Alzheimer's disease

Parkinson's disease

\section{ABSTRACT}

Cognitive dysfunction and dementia are important causes of disability and mortality in the elderly population. Over a decade, many studies have found evidence that greater arterial stiffness is associated with impaired cognitive function. Stiff arteries have a direct effect on the brain microvasculature. In this review, we present evidence that arterial stiffness is linked to cognitive dysfunction in neurodegenerative disorders represented by Alzheimer's disease and Parkinson's disease. We also discuss possible mechanisms of arterial stiffness and cognitive dysfunction other than the universal pathologic features of beta-amyloid for Alzheimer's disease and alpha-synuclein for Parkinson's disease.

\section{Introduction}

Cognitive impairment and dementia are common in the elderly and show increasing prevalence in the current society with the growing proportion of people of advanced age. Cognitive impairment is associated with increased deficits in activities of daily living ${ }^{1}$ and is also an important predictor of morbidity and mortality ${ }^{2}$. Vascular risk factors including hypertension, diabetes mellitus, and dyslipidemia have a potent role in cognitive impairment and dementia ${ }^{2-4}$.

Arterial stiffness is a marker of vascular aging and dysfunction ${ }^{5}$ and a strong predictor of cardiovascular diseases and mortality ${ }^{6}$. Many investigations have suggested that arterial stiffness is a predictor and risk factor of cognitive impairment and dementia ${ }^{7-26}$. The aim of this study is to review the relationship between arterial stiffness and cognitive impairment in neurodegenerative diseases including Alzheimer's disease (AD) and Parkinson's disease (PD).

\section{Arterial stiffness}

The arterial system starts in the heart and terminates at the peripheral microvascular flow ${ }^{27}$. The proximal large artery has elasticity and absorbs blood flow pressure from the heart, reducing blood flow pressure in the peripheral artery and end organ ${ }^{27}$. The distensibility of the artery is inversely related to the arterial pulse wave: increased pulse wave velocity (PWV) is associated with a stiffer artery ${ }^{28}$. As the artery becomes older, its cushioning function is decreased and the stiffer artery leads to reduced absorption of blood flow pulsatility, resulting in pulsatile flow to organs with a high oxygen demand such as the brain and kidneys ${ }^{27}$. Arterial stiffness causes the pulse wave to flow faster and increases left ventricular load, leading to left ventricular dysfunction and cardiac failure ${ }^{27}$. Reduced diastolic aortic pressure decreases the coronary artery perfusion pressure and causes myocardial ischemia ${ }^{27}$. 
Increased pulsation due to a stiffened artery enters into the microvascular system and predisposes arterial walls to rupture, resulting in the development of microinfarcts and microhemorrhages in brain and kidneys ${ }^{27}$.

Measurement of PWV is generally accepted as the most simple, non-invasive, robust, and reproducible method to determine arterial stiffness ${ }^{29}$. The brachial artery to ankle (baPWV) and carotid artery to femoral artery (cfPWV) are most widely used methods ${ }^{28}$. Central pulse wave analysis and augmentation index are other indirect methods to measure arterial stiffness ${ }^{29}$. However, PWV is a precise and simple test and has been used most widely in studies of arterial stiffness and cognitive impairment.

Atrial stiffness and cognitive dysfunction in Alzheimer's disease

Many cross-sectional studies have reported the association between arterial stiffness and cognitive function. Atrial stiffness was associated with subjective memory impairment, mild cognitive impairment (MCI), and dementia ${ }^{26}$. A previous study of 84 subjects found that $\mathrm{CPPWV}$ was inversely correlated with results of the Mini Mental Status Examination (MMSE) ${ }^{7}$. In 352 elderly communitydwelling individuals, the middle tertile of baPWV was an independent risk factor for a low MMSE score (odds ratio $=9.66,95 \%$ confidence interval $(\mathrm{CI})=1.15-80.93)^{8}$. Other authors studied 308 elderly subjects with memory impairment, who were categorized into $\mathrm{MCI}, \mathrm{AD}$, vascular dementia, and normal cognition ${ }^{9}$. PWV was significantly higher in subjects with vascular dementia or AD than in those with normal cognition $(\mathrm{p}<0.0001)$ and PWV was higher in subjects with MCI than in those with normal cognition ( $\mathrm{p}=$ 0.01) [9]. A community-based study with 203 subjects found that baPWV was significantly increased in the low MMSE score group $(\mathrm{p}=0.003)^{10}$. A large population-based study with 3,714 subjects demonstrated that increased cfPWV was associated with worse performance on the Stroop test $(\beta=-1.13 ; 95 \% \mathrm{CI}=0.26 ; 1.99)^{12}$; however, $\mathrm{PWV}$ was not associated with MMSE, letter digit substitution task, and word fluency test $\mathrm{t}^{12}$. In a study of 110 non-diabetic, nevertreated patients with essential hypertension, increased cfPWV was associated with low MMSE score $(0.36 ; \mathrm{p}=$ $0.026)^{13}$. A re-designed cross-sectional study with 409 patients was conducted with detailed cognitive tests including visuospatial organization (block design, object assembly, visual reproductions immediate and delayed, Hooper visual organization test, and matrix reasoning), scanning and tracking (trail making tests, digit symbol substitution, and symbol search), verbal episodic memory (immediate and delayed Hopkins verbal learning test), working memory (digit span forward and backward, letternumber sequence, and controlled oral word associations), and global composite score ${ }^{15}$. Carotid-femoral PWV was associated with worse performance on visuospatial organization, verbal memory, and global cognitive function $(\mathrm{p}<0.05)^{15}$. An increase in PWV with age was also associated with a decrease in cognitive performance ${ }^{15}$. One substudy with 552 participants showed similar results ${ }^{16}$. After adjusting for other confounding factors, increased PWV had association with worse score in MMSE $(\beta=-0.55$; $95 \%$ CI $=-0.91 ;-0.19$ ), psychomotor speed (boxes and digit copying test, $\beta=-1.59 ; 95 \% \mathrm{CI}=-3.03 ;-0.15)$, and perceptual speed (pattern and letter comparison test, $\beta=$ $-0.60 ; 95 \% \mathrm{CI}=-0.98 ;-0.22$ ), but no association with verbal memory (Buschke selective reminding test, $\beta=-0.20 ; 95 \%$ $\mathrm{CI}=-0.44 ; 0.04)^{16}$. One community-based study showed no association between PWV and cognitive function; of 319 participants aged 70-90 years, cfPWV had no significant association with indicators of cognitive function including processing speed (digit symbol coding and trail making test A), memory (logical memory Story A , Rey auditory visual verbal learning test, and the Benton visual retention test), language (animal naming and the 30-item Boston naming test), executive function (phonemic fluency, trail making test B, and the Stroop test), visuospatial function (block design), and global composite score ${ }^{19}$. In another large cohort of older adults, 1,436 participants were assessed for MMSE score, executive function, attention, speed, memory, and semantic memory ${ }^{20}$. High cfPWV was associated with worse performance on the MMSE $(\beta=-0.31 ; p=0.005)$, Rey auditory verbal learning test $(\beta=-1.10 ; p=0.01)$, and the composite cognition score $(\beta=-0.10 ; p=0.04)$, whereas carotid-radial PWV had no association with cognitive dysfunction ${ }^{20}$. A recent community-based cohort study with 1,101 subjects showed the risk associated with arterial stiffness $^{22}$. Higher continuous cfPWV increased the risk of MCI ( $\mathrm{p}<0.03)$, but not all-cause dementia and Alzheimer's disease $^{22}$. Arterial stiffness was an independent predictor of $\mathrm{MCI}^{22}$. Another recent study revealed that higher arterial stiffness was associated with $\mathrm{MCI}^{23}$. In 4,461 subjects, the highest quartile of cfPWV was associated with a greater prevalence of MCI than the lowest quartile of cfPWV in white individuals (odds ratio $=1.27 ; 95 \% \mathrm{CI}=1.02 ; 1.56)^{23}$. Arterial stiffness was associated with cognitive dysfunction in patients with subjective memory complaints. In 198 elderly patients with hypertension, cfPWV was the only significant predictor for memory impairment of men (odds ratio $=1.22 ; 95 \% \mathrm{CI}=1.00 ; 1.49, \mathrm{p}=0.05)^{24}$. Higher $\mathrm{cfPWV}$, normalized for mean blood pressure, was significantly associated with a lower MMSE score $(\mathrm{p}<0.05)$ in 280 subjects with subjective memory complaints ${ }^{25}$. Our recent study also showed that baPWV in subjects with subjective memory impairment was higher than healthy controls, although not statistically insignificant $(\mathrm{p}=0.058)^{26}$.

Several longitudinal studies have investigated the association between arterial stiffness and cognitive function. Most of these studies revealed that arterial stiffness was a potent predictor or risk factor for MCI 
or dementia. In one study, during a mean follow-up of 4.4 years no associations were found between PWV and cognitive decline or risk of dementia ${ }^{11}$. In another longitudinal study of 102 elderly subjects with 12 months of follow-up, cfPWV was related to a greater annual decline in MMSE score $(\beta=-0.736 ; p<0.001)^{12}$. The authors concluded that PWV was a strong predictor of cognitive dysfunction ${ }^{12}$. In addition, a longitudinal study performed over 14 years with 582 subjects revealed that PWV and cognitive decline were related ${ }^{13}$. Subjects with higher baseline cfPWV exhibited a prospective decline in verbal learning (California verbal learning test), delayed recall (California verbal learning test), nonverbal memory test (Benton visual retention test), and general cognitive screening test $(p<0.05)^{13}$. Moreover, another study analyzed the 1-year cognitive decline in nursing home patients with dementia who were older than 80 years ${ }^{17}$. Among 873 patients, increased cfPWV was associated with a greater decrease in MMSE $(p<0.03)^{17}$. A communitybased study with 1,559 subjects demonstrated that higher cfPWV was not associated with memory (logical memorydelayed recall), and executive functions (trail making test part B minus part A score); however, increased cfPWV was associated with lower total cerebral brain volume, which is found in Alzheimer's disease $(\mathrm{p}<0.05)^{18}$. A recent 5 -year follow-up study was performed in community dwelling older adults ${ }^{21}$. The odds ratios for cognitive decline were higher in the highest tertile $(2.95 ; 95 \% \mathrm{CI}=1.29 ; 6.74)$ and middle tertile $(2.39 ; 95 \% \mathrm{CI}=1.11 ; 5.15)$ of baPWV, compared with the lowest tertile ${ }^{21}$. Therefore, high baPWV was an independent predictor of cognitive dysfunction ${ }^{21}$.

However, the pathophysiologic mechanisms linking arterial stiffness and cognitive dysfunction have not been determined. As stiffened artery causes loss of cushioning capacity, the increased pulsatile pressure and flow leads to increased vascular resistance and small vessel damage $\mathrm{e}^{30,31}$. The accumulated brain damage results in brain atrophy and cognitive dysfunction ${ }^{30,31}$. Other suggested etiologies include heart diastolic dysfunction ${ }^{32}$, medial temporal lobe atrophy ${ }^{33}$, reduced white matter integrity ${ }^{34}$, increased white matter hyperintensities ${ }^{35}$, progression of betaamyloid deposition $^{36}$, and impaired renal function ${ }^{26}$.

Arterial stiffness and cognitive dysfunction in Parkinson's disease

There are several reports on arterial stiffness in PD. In one study, measurement of cardio-ankle vascular stiffness index using $\mathrm{PWV}^{37}$ in $63 \mathrm{PD}$ subjects showed no difference in cardio-ankle vascular stiffness index compared with normal controls ${ }^{37}$. Another study found that arterial stiffness was associated with cardiovascular autonomic dysfunction in patients with $\mathrm{PD}^{36}$. Greater arterial stiffness was associated with orthostatic hypotension, supine hypertension, nocturnal hypertension, and nondipping ${ }^{38}$.
A study including 27 patients with PD and five patients with multiple system atrophy showed that mean PWV at night was significantly elevated in patients compared with controls $(p=0.04)^{39}$. In addition, cognitive dysfunction was related to neurocirculatory abnormalities ${ }^{40}$. Cognitive impairment was associated with a reduction in blood pressure during orthostasis and an increase in systolic pressure while supine, and these blood pressure abnormalities were associated with elevated white matter hyperintensities ${ }^{40}$. Arterial stiffness leads to cardiovascular autonomic abnormalities and causes white matter hyperintensities in brain, and therefore may be associated with cognitive dysfunction in PD.

We recently studied an association between arterial stiffness and cognitive dysfunction in patients with Lewy body disorder (LBD). In LBD patients, higher baPWV was associated with lower MMSE score $(\beta \pm$ SE $=-0.003 \pm$ $0.001, p=0.007$ ) and more severe dementia. Higher baPWV was also associated with lower performance in attention (digit span test), language (Korean Boston naming test), visuospatial function (Rey complex figure test), memory (Seoul verbal learning test), and executive function (control oral word association test) in LBD patients. This suggests that vascular brain injury is associated with cognitive dysfunction in $\mathrm{LBD}^{41}$.

\section{Conclusion}

This brief review highlights consistent findings that greater arterial stiffness is associated with cognitive dysfunction in normal elderly people, patients with subjective memory impairment, patients with MCI, and patients with AD. This supports the hypothesis that greater arterial stiffness has a potent role in microvascular brain damage and subsequent brain atrophy. However, strong evidence linking arterial stiffness and AD is lacking. Further longitudinal study is needed to investigate the effect of arterial stiffness on the development of AD. In addition, the role of arterial stiffness on cognitive dysfunction in PD should be further investigated.

\section{References}

1. Gill TM, Richardson ED, Tinetti ME. Evaluating the risk of dependence in activities of daily living among community-living older adults with mild to moderate cognitive impairment. J Gerontol A Biol Sci Med Sci. 1995; 50(5): M235-241.

2. Gorelick PB, Scuteri A, Black SE, et al. Vascular contributions to cognitive impairment and dementia: a statement for healthcare professionals from the american heart association/american stroke association. Stroke. 2011; 42(9): 2672-2713.

3. de la Torre JC. Cardiovascular risk factors promote brain hypoperfusion leading to cognitive decline and dementia. Cardiovascular psychiatry and neurology. 2012;2012:367516.

4. Picano E, Bruno RM, Ferrari GF, et al. Cognitive impairment and cardiovascular disease: so near, so far. Int J Cardiol. 2014; 175(1): 21-29.

5. Singer J, Trollor JN, Baune BT, et al. Arterial stiffness, the brain and cognition: a systematic review. Ageing Res Rev. 2014; 15: 16-27. 
6. Vlachopoulos C, Aznaouridis K, Stefanadis C. Prediction of cardiovascular events and all-cause mortality with arterial stiffness: a systematic review and meta-analysis. J Am Coll Cardiol. 2010; 55(13): 1318-1327.

7. Scuteri A, Brancati AM, Gianni W, et al. Arterial stiffness is an independent risk factor for cognitive impairment in the elderly: a pilot study. J Hypertens. 2005; 23(6): 1211-1216.

8. Fujiwara Y, Chaves PH, Takahashi R, et al. Arterial pulse wave velocity as a marker of poor cognitive function in an elderly community-dwelling population. J Gerontol A Biol Sci Med Sci. 2005; 60(5): 607-612.

9. Hanon $\mathrm{O}$, Haulon $\mathrm{S}$, Lenoir $\mathrm{H}$, et al. Relationship between arterial stiffness and cognitive function in elderly subjects with complaints of memory loss. Stroke. 2005; 36(10): 2193-2197.

10. Fukuhara M, Matsumura K, Ansai T, et al. Prediction of cognitive function by arterial stiffness in the very elderly. Circ J. 2006; 70(6): 756-761.

11. Poels MM, van Oijen M, Mattace-Raso FU, et al. Arterial stiffness, cognitive decline, and risk of dementia: the Rotterdam study. Stroke. 2007; 38(3): 888-892.

12. Scuteri A, Tesauro M, Appolloni S, et al. Arterial stiffness as an independent predictor of longitudinal changes in cognitive function in the older individual. J Hypertens. 2007; 25(5): 1035-1040.

13. Waldstein SR, Rice SC, Thayer JF, et al. Pulse pressure and pulse wave velocity are related to cognitive decline in the Baltimore Longitudinal Study of Aging. Hypertension. 2008; 51(1): 99-104.

14. Triantafyllidi $\mathrm{H}$, Arvaniti C, Lekakis J, et al. Cognitive impairment is related to increased arterial stiffness and microvascular damage in patients with never-treated essential hypertension. Am J Hypertens. 2009; 22(5): 525-530.

15. Elias MF, Robbins MA, Budge MM, et al. Arterial pulse wave velocity and cognition with advancing age. Hypertension. 2009; 53(4): 668-673.

16. Watson NL, Sutton-Tyrrell K, Rosano C, et al. Arterial stiffness and cognitive decline in well-functioning older adults. J Gerontol A Biol Sci Med Sci. 2011; 66(12): 1336-1342.

17. Benetos A, Watfa G, Hanon 0 , et al. Pulse wave velocity is associated with 1-year cognitive decline in the elderly older than 80 years: the PARTAGE study. J Am Med Dir Assoc. 2012; 13(3): 239-243.

18. Tsao CW, Seshadri S, Beiser AS, et al. Relations of arterial stiffness and endothelial function to brain aging in the community. Neurology. 2013; 81(11): 984-991.

19. Singer J, Trollor JN, Crawford J, et al. The association between pulse wave velocity and cognitive function: the Sydney Memory and Ageing Study. PLoS One. 2013; 8(4): e61855.

20. Zhong W, Cruickshanks KJ, Schubert CR, et al. Pulse wave velocity and cognitive function in older adults. Alzheimer Dis Assoc Disord. 2014; 28(1): 44-49.

21. Taniguchi Y, Fujiwara Y, Nofuji Y, et al. Prospective Study of Arterial Stiffness and Subsequent Cognitive Decline Among CommunityDwelling Older Japanese. J Epidemiol. 2015; 25(9): 592-599.

22. Pase MP, Beiser A, Himali JJ, et al. Aortic Stiffness and the Risk of Incident Mild Cognitive Impairment and Dementia. Stroke. 2016; 47(9): 2256-2261

23. Meyer ML, Palta P, Tanaka H, et al. Association of Central Arterial Stiffness and Pressure Pulsatility with Mild Cognitive Impairment and Dementia: The Atherosclerosis Risk in Communities StudyNeurocognitive Study (ARIC-NCS). Journal of Alzheimer's disease : JAD. 2017; 57(1): 195-204.
24. Kearney-Schwartz A, Rossignol P, Bracard S, et al. Vascular structure and function is correlated to cognitive performance and white matter hyperintensities in older hypertensive patients with subjective memory complaints. Stroke. 2009; 40(4): 1229-1236.

25. Scuteri A, Tesauro M, Guglini L, et al. Aortic stiffness and hypotension episodes are associated with impaired cognitive function in older subjects with subjective complaints of memory loss. Int J Cardiol. 2013; 169(5): 371-377.

26. Oh YS, Kim JS, Park JW, et al. Arterial stiffness and impaired renal function in patients with Alzheimer's disease. Neurol Sci. 2016; 37(3): 451-457.

27. Adji A, O’Rourke MF, Namasivayam M. Arterial stiffness, its assessment, prognostic value, and implications for treatment. Am J Hypertens. 2011; 24(1): 5-17.

28. Rabkin SW. Arterial stiffness: detection and consequences in cognitive impairment and dementia of the elderly. Journal of Alzheimer's disease : JAD. 2012; 32(3): 541-549.

29. Laurent S, Cockcroft J, Van Bortel L, et al. Expert consensus document on arterial stiffness: methodological issues and clinical applications. Eur Heart J. 2006; 27(21): 2588-2605.

30. Zeki Al Hazzouri A, Yaffe K. Arterial stiffness and cognitive function in the elderly. Journal of Alzheimer's disease : JAD. 2014; 42 Suppl 4: S503-514.

31. van Sloten TT, Protogerou AD, Henry RM, et al. Association between arterial stiffness, cerebral small vessel disease and cognitive impairment: A systematic review and meta-analysis. Neurosci Biobehav Rev. 2015; 53: 121-130.

32. Calik AN, Ozcan KS, Yuksel G, et al. Altered diastolic function and aortic stiffness in Alzheimer's disease. Clin Interv Aging. 2014; 9: $1115-1121$.

33. Lilamand M, Vidal JS, Plichart M, et al. Arterial stiffness and medial temporal lobe atrophy in elders with memory disorders. J Hypertens. 2016; 34(7): 1331-1337.

34. Maillard P, Mitchell GF, Himali JJ, et al. Effects of Arterial Stiffness on Brain Integrity in Young Adults From the Framingham Heart Study. Stroke. 2016; 47(4): 1030-1036.

35. Saji N, Ogama N, Toba K, et al. White matter hyperintensities and geriatric syndrome: An important role of arterial stiffness. Geriatr Gerontol Int. 2015; 15 Suppl 1: 17-25.

36. Hughes TM, Craft S, Lopez OL. Review of 'the potential role of arterial stiffness in the pathogenesis of Alzheimer's disease'. Neurodegener Dis Manag. 2015; 5(2): 121-135.

37. Suzuki J, Sakakibara R, Tateno F, et al. Parkinson's disease and the cardio-ankle vascular stiffness index. Intern Med. 2014; 53(5): 421426.

38. Kim JS, Lee SH, Oh YS, et al. Arterial Stiffness and Cardiovascular Autonomic Dysfunction in Patients with Parkinson's Disease. Neurodegener Dis. 2017; 17(2-3): 89-96.

39. Franzen K, Fliegen S, Koester J, et al. Central hemodynamics and arterial stiffness in idiopathic and multiple system atrophy. J Neurol. 2017; 264(2): 327-332.

40. Kim JS, Oh YS, Lee KS, et al. Association of cognitive dysfunction with neurocirculatory abnormalities in early Parkinson disease. Neurology. 2012; 79(13): 1323-1331.

41. Ryu DW, Kim JS, Lee JE, et al. Association of arterial stiffness with cognition in patients with Lewy body disorder. Neurol Sci. 2017; doi: 10.1007/s10072-017-2977-7. 